\title{
SHORT REPORT \\ Discontinuation of contact precautions with the introduction of universal daily chlorhexidine bathing
}

\author{
J. A. MCKINNELL ${ }^{1,2,3 *}$, S. J. EELLS ${ }^{2,4,5}$, E. CLARK ${ }^{1}$, D. D. RAND ${ }^{1}$, \\ G. T. KIET ${ }^{1}$, R. MACIAS-GIL ${ }^{2}$, J. M. MENDEZ ${ }^{2,3}$, S. S. HUANG ${ }^{6}$, \\ E. N. MILEFCHIK ${ }^{1}$ AND L. G. MILLER MI $^{2,3}$ \\ ${ }^{1}$ Torrance Memorial Medical Center, Torrance, California, USA \\ ${ }^{2}$ Division of Infectious Disease, Los Angeles Biomedical Research Institute, Torrance, California, USA \\ ${ }^{3}$ David Geffen School of Medicine at UCLA, Los Angeles, California, USA \\ ${ }_{5}^{4}$ Department of Epidemiology, UCLA Fielding School of Public Health, Los Angeles, California, USA \\ ${ }^{5}$ Science 37, Inc., Los Angeles, California, USA \\ ${ }^{6}$ Division of Infectious Diseases and Health Policy Research Institute, University of California, Irvine School of \\ Medicine, Irvine, California, USA
}

Received 10 October 2016; Final revision 11 May 2017; Accepted 13 May 2017; first published online 9 June 2017

\section{SUMMARY}

Contact precautions are a traditional strategy to prevent transmission of methicillin-resistant Staphylococcus aureus (MRSA). Chlorhexidine bathing is increasingly used to decrease MRSA burden and transmission in intensive care units (ICUs). We sought to evaluate a hospital policy change from routine contact precautions for MRSA compared with universal chlorhexidine bathing, without contact precautions. We measured new MRSA acquisition in ICU patients and surveyed for MRSA environmental contamination in common areas and non-MRSA patient rooms before and after the policy change. During the baseline and chlorhexidine bathing periods, the number of patients (453 vs. 417), ICU days (1999 vs. 1703) and MRSA days/1000 ICU days (109 vs. 102) were similar. MRSA acquisition (2/453 vs. $2 / 457, P=0.93)$ and environmental MRSA contamination $(9 / 474$ vs. $7 / 500, P=0.53)$ were not significantly different between time periods. There were $58 \%$ fewer contact precaution days in the ICU during the chlorhexidine period $(241 / 1993 v s .102 / 1730, P<0 \cdot 01)$. We found no evidence that discontinuation of contact precautions for patients with MRSA in conjunction with adoption of daily chlorhexidine bathing in ICUs is associated with increased MRSA acquisition among ICU patients or increased MRSA contamination of ICU fomites. Although underpowered, our findings suggest this strategy, which has the potential to reduce costs and improve patient safety, should be assessed in similar but larger studies.

Key words: Chlorhexidine, contact precautions, hospital epidemiology, hygeine and hospital infections, infection prevention, MRSA, VRE.

\footnotetext{
* Author for correspondence: J. A. McKinnell, 1000 West Carson Street, Box 466, Torrance, CA, USA.

(Email: Dr.McKinnell@gmail.com)
}

\section{INTRODUCTION}

The use of contact precautions for patients with methicillin-resistant Staphylococcus aureus (MRSA) colonisation or infection is considered standard practice in most hospitals in the United States. The 
Centers for Disease Control and Prevention, the Infectious Disease Society of America, and the Society for Healthcare Epidemiology all recommend contact precautions for patients with MRSA [1-3]. Despite its widespread use, the evidence for the effectiveness of contact precautions for prevention of MRSA infections in non-outbreak settings is limited [4]. A randomised trial found that active surveillance for MRSA increased contact precautions usage, but had no impact on MRSA transmission [5].

Contact precautions have limitations. While there are conflicting estimates for the unintended impact of contact precautions [6], such measures have been associated with fewer healthcare worker visits, lower patient satisfaction, and more preventable adverse events including falls, decubitus ulcers and hypoglycaemia [7-9]. Implementation of contact precautions is labour and cost-intensive [4].

Contact precautions theoretically prevent infection by limiting the opportunity for MRSA acquisition by other patients. MRSA-infected patients are known to contaminate the hospital environment, serving as a source of potential transmission to healthcare workers, visitors, and other patients [10]. However, recent data using whole genome sequencing suggest that only $20 \%$ of MRSA acquisitions in the intensive care unit (ICU) can be attributed to patient-to-patient transmission [11].

Another means to prevent MRSA acquisition is daily chlorhexidine bathing. Large randomised trials of universal chlorhexidine bathing in ICUs support the effectiveness of universal chlorhexidine combined with contact precautions in the prevention of MRSA acquisition and hospital acquired infections [12-14]. Universal chlorhexidine-based body decolonisation has an advantage over contact precautions in that chlorhexidine reduces total body burden of MRSA carriers and has a prolonged antiseptic effect that protects non-carries from MRSA acquisition [15].

Multiple medical centres have discontinued MRSA contact precautions after chlorhexidine bathing has been introduced, but little published data exist on this strategy. Our current investigation was designed to evaluate a change in hospital policy of discontinuing contact precautions in the presence of universal chlorhexidine bathing.

\section{METHODS}

We evaluated the impact of a hospital policy to discontinue contact precautions for MRSA after the introduction of universal chlorhexidine bathing in terms of environmental contamination and patient acquisition of MRSA over a 6-month period. We performed our study at Torrance Memorial Medical Center, a 377-bed community hospital located in Torrance, California. Chlorhexidine bathing for hospitalised patients had not been used in the hospital prior to the intervention, except as routine preoperative preparation. The hospital includes three ICUs: 22-bed Medical-Surgical unit (MICU), eight-bed Burn ICU (BICU), and eight-bed Cardiac ICU (CICU). The average nursing-to-patient ratio in the ICU was 2 to 1 . All patients are treated in single rooms. The study was conducted as a quality improvement investigation and was designated as an exempt quality improvement project by the Torrance Memorial Medical Center Institutional Review Board.

\section{Patient surveillance and contact precautions}

By California state legislative mandate, all patients admitted to an ICU must undergo admission surveillance for MRSA. Surveillance is conducted within $24 \mathrm{~h}$ of admission or transfer, typically within $8 \mathrm{~h}$. The nares is the only body site tested for MRSA. Clinical cultures are collected as a matter of routine care. Torrance Memorial Medical Center also conducts MRSA surveillance for all patients discharged from the ICU. Surveillance swabs are collected by the ICU nursing staff and undergo PCR-based testing for MRSA (Cepheid, GeneXpert, Sunnyvale, CA).

Contact precautions consist of donning gowns and gloves. There are no anterooms for donning gowns and gloves. Staff are instructed to don gown and gloves in the hall prior to entering patient rooms. Hand hygiene is also required before and after patient contact. Alcohol-based hand gels may be used for hand hygiene with the exception of cases of Clostridium difficile where soap and water is specified. During the baseline period, hospital policy dictated that patients were placed in contact precautions for either (1) MRSA or (2) 'Enteric pathogens', defined as $C$. difficile, vancomycin-resistant Enterococcus faecium (VRE), carbapenem-resistant Enterobacteriaceae, carbapenem-resistant Pseudomonas spp., and carbapenem-resistant Acinetobacter spp.

\section{Intervention}

Traditionally, all ICU patients received routine daily bed baths with non-medicated soap and water as directed by hospital policy. The routine bathing 
period occurred during February and March. During the chlorhexidine period, all patients were bathed daily with $4 \%$ chlorhexidine gluconate from the neck down. Nurses were instructed on chlorhexidine bathing for 8 weeks prior to initiation of chlorhexidine during April and May. During the chlorhexidine period during June and July, hospital policy was modified to no longer require that patients with MRSA colonisation or infection have to be placed in contact precautions.

\section{Infection surveillance and patient outcome evaluation}

The Torrance Memorial Medical Center Department of Infection Control maintains a programme of infection surveillance for all ICU patients. MRSA surveillance is conducted on admission and discharge using nares swabs. MRSA acquisition is defined when non-MRSA carriers, negative surveillance screen and no clinical cultures for MRSA within the first $72 \mathrm{~h}$, are subsequently found to carry MRSA on discharge surveillance or clinical cultures collected $>72 \mathrm{~h}$ after ICU admission.

All ICU patients are monitored for acquisition of nosocomial infection using current National Healthcare Safety Network guidelines [16]. In addition, medical records for all cases of ICU mortality were reviewed by an investigator (J.A.M.) to assess death associated with sepsis or documented infection. Nursing hand hygiene compliance is measured in the largest ICU (MICU) on a monthly basis with covert observations ('secret-shoppers'). Data on ICU mortality and infectious outcomes of ICU stay were also collected.

\section{Environmental cleaning and surveillance}

Hospital policy requires the Environmental Services department at Torrance Memorial Medical Center to clean every occupied room and common work surface in the ICU every day with a quaternary ammonium chloride compound (Ecolabs, St. Paul, MN). Routine cleaning starts in the morning and continues during the regular workday. In addition to routine cleaning, Environmental Services can be called for specific cleaning needs. The effectiveness of environmental cleaning is routinely monitored with adenosine tri-phosphate luminescence to measure organic matter on environmental surfaces (3M Clean-Trace Hygiene Management System, St. Paul, MN). Routine cleaning by Environmental Services does not include patient care items, including IV poles, ICU monitor wires.
Patient care items are cleaned by nursing staff at the start of the regular workday.

Environmental surveillance culturing was conducted in each ICU three times per week for 8 weeks prior to policy change and 8 weeks after policy change. Surveillance was conducted in the morning prior to routine cleaning. High-touch common workspace and patient room surfaces were prospectively defined prior to start of the study based on routine workflow habits of physicians, nurses, and other hospital staff. The common high-touch workspace surfaces included five workspace counters frequently used for documentation (three MICU, one BICU, one $\mathrm{CICU}$ ) and 15 high-use computer keyboards (nine MICU, three BICU, three CICU). The hightouch patient room surfaces tested in our study included the bedrails, vitals monitor, intravenous pumps and the bedside table based on evidence from the literature [17]. We performed in-room surveillance for non-MRSA patient rooms, i.e. negative admission surveillance and no positive clinical cultures to date. Whenever possible, rooms were chosen with a nurse who was also seeing an MRSA colonised or infected patient. If a shared nurse could not be identified, rooms adjacent to an MRSA carrier were chosen. Ten non-MRSA patient rooms were sampled on each sampling day.

After collection, the environmental samples were transported promptly to a research laboratory and placed in a Stomacher (Seward, Worthing, West Sussex, UK) to homogenise samples. The sample was then enriched in trypticase soy broth with $7 \%$ sodium chloride (BD, East Rutherford, NJ) and incubated overnight at $37^{\circ} \mathrm{C}$. The culture broth was plated onto BBL CHROMagar MRSA media (BD, East Rutherford, NJ) and incubated for $24 \mathrm{~h}$ at $37^{\circ} \mathrm{C}$. Positive isolates were confirmed as $S$. aureus by positive catalase and Staphaurex tests (Remel, Lenexa, Kansas).

\section{Data analyses}

The study duration was defined based on the time available to conduct the study prior to the planned change in hospital policy. Infection and colonisation data were collected for 3 months prior to the policy change and 3 months following the policy change. Further data collection was not possible as ICU services were moved to a new hospital tower. Data were analysed using SAS (ver. 9.3; SAS Institute, Cary, NC). The $\chi^{2}$ tests and $t$-tests were performed as appropriate and 
associated $P$ values calculated to assess factors associated with each time period. The $95 \%$ confidence limits were calculated for the environmental contamination and the difference in contamination for each time period. All variables were considered significant at the $\alpha=0.05$ level.

\section{RESULTS}

During the period where contact precautions were applied to MRSA carriers, 453 patients were admitted to ICU and spent a total of 1993 patient days in ICU. During the chlorhexidine period where contact precautions were stopped for MRSA, 417 patients were admitted and spent a total of 1730 patient days in the ICU. There were no adverse events noted with the use of chlorhexidine during the chlorhexidine period. Nursing hand hygiene compliance was $94 \%$ during the baseline period and $86 \%$ during the chlorhexidine period but hand hygiene of doctors and non-nursing staff was not available. The effectiveness of environmental cleaning as routinely monitored with adenosine tri-phosphate was $84 \%$ in CICU, $95 \%$ in MICU, and $93 \%$ in BICU, and did not differ during the baseline and chlorhexidine periods.

The number of patients with MRSA on their admission screen was similar in the two periods $(6 \cdot 3 \% v s$. $6.0 \%, P=0.86$ ) as were the number of patients with a history of MRSA $(8 \cdot 0 \%$ vs. $8 \cdot 6 \%, P=0 \cdot 71)$, admission clinical culture with MRSA ( $1 \cdot 8 \%$ vs. $1 \cdot 7 \%, P=0.92)$ and the number of MRSA patient days/1000 ICU patient days was similar (109 vs. 102, $P=0 \cdot 57$ ).

Thirty-one patients with MRSA colonisation who would have been placed in contact precautions were not placed in isolation during the chlorhexidine only period. There were $58 \%$ fewer contact precaution days during the chlorhexidine period compared with the baseline period $(241 / 1993$ vs. $102 / 1730, P<0 \cdot 01)$. The number of contact precaution days for MRSA was lower during the chlorhexidine period $(218 / 1993$ vs. $0 / 1730, P<0 \cdot 01$ ), and there was a statistically significant increase in enteric pathogen-associated isolation days $(23 / 1993$ vs. 102/1736, $P<0 \cdot 01)$.

There were $9 / 474(1.9 \%)$ positive MRSA environmental cultures in the contact precautions period (three adjacent rooms, six common areas) and 7/500 $(1 \cdot 4 \%)$ MRSA contamination events in the chlorhexidine period (two adjacent rooms, five common areas $P=0.53$ ). The difference in MRSA environmental contamination between the chlorhexidine and contact precautions periods was $-0 \cdot 5 \%,(95 \%$ C.I. $=-2 \cdot 3$ to
$1 \cdot 3 \%$ ). There were two MRSA acquisition events in the contact precaution and two MRSA acquisition events in the chlorhexidine period $(0 \cdot 4 \%$ vs. $0.5 \%$, $P=0.93)$.

ICU mortality was higher in the contact precautions period than the chlorhexidine period $(11.5 \% \mathrm{vs}$. $6 \cdot 5 \%, P=0 \cdot 01)$. There was a non-significant trend towards higher infection/sepsis-associated mortality in the contact precautions period $(2 \cdot 4 \%$ vs. $1 \cdot 7 \%$, $P=0 \cdot 12$ ). Further details of patient outcomes are provided in Table 1.

\section{DISCUSSION}

We evaluated a single centre hospital policy change that consisted of discontinuing contact precautions after the initiation of universal chlorhexidine bathing. This community hospital was notable for high rates of compliance with hand hygiene and environmental cleaning. We found no evidence for a difference in environmental contamination with MRSA in the chlorhexidine period when contact precautions were discontinued compared with the baseline period when MRSA carriers were placed in isolation. The number of MRSA acquisition events was also similar. The results from our investigation should not be considered definitive based on our small sample size, limited study duration, study design, and single centre design. However, our findings highlight important considerations for hospitals considering a similar policy change and highlight the need for large scale properly conducted clinical trials.

Indeed, our results demonstrate that the discontinuation of contact precautions during the chlorhexidine period was associated with a large reduction in contact precaution days. This reduction and the associated costs to the medical centre represents an important financial benefit to hospitals if the approach described above can be established to be safe for ICU patients [4].

It is plausible that chlorhexidine bathing may be effective enough to allow for discontinuation of contact precautions as chlorhexidine bathing may decrease MRSA bioburden on ICUs patients, which, in turn, decreases the likelihood of MRSA transmission and environmental contamination [12-14]. Our results are encouraging that chlorhexidine bathing may indeed be effective enough to obviate the need for contact precautions.

Our study was limited in duration based on a relatively quick decision to change hospital policy and an 
Table 1. ICU characteristics during contact precautions compared with universal chlorhexidine

\begin{tabular}{|c|c|c|c|}
\hline & $\begin{array}{l}\text { Contact precautions } \\
N=453(\%)\end{array}$ & $\begin{array}{l}\text { Universal CHG } \\
N=417(\%)\end{array}$ & $P$ Value \\
\hline \multicolumn{4}{|l|}{ Admission characteristics and MRSA colonisation pressure } \\
\hline Admission screen MRSA+ & $26(6 \cdot 3)$ & $24(6 \cdot 0)$ & $0 \cdot 86$ \\
\hline History of MRSA & $36(8 \cdot 0)$ & $36(8 \cdot 6)$ & $0 \cdot 71$ \\
\hline MRSA+ clinical culture at admission & $8(1 \cdot 8)$ & $7(1 \cdot 7)$ & $0 \cdot 92$ \\
\hline ICU patient days & 1993 & 1730 & \\
\hline Average ICU length of stay, mean \pm s.D., median, (range) & $\begin{array}{l}4 \cdot 4 \text { days } \pm 5 \cdot 4 \text { days } \\
3 \cdot 0,(1 \cdot 0-47)\end{array}$ & $\begin{array}{l}4 \cdot 15 \text { days } \pm 3 \cdot 8 \text { days } \\
3 \cdot 0,(1 \cdot 0-38)\end{array}$ & $0 \cdot 43$ \\
\hline MRSA patient days & 218 & 178 & $0 \cdot 52$ \\
\hline Contact precaution days & 241 & 102 & $<0 \cdot 01$ \\
\hline Enteric pathogen (VRE, $C$. diff) contact precaution days & 23 & 102 & $<0 \cdot 01$ \\
\hline MRSA contact precaution days & 218 & 0 & $<0 \cdot 01$ \\
\hline MRSA patient days/1000 ICU patient days & 109 & 102 & $0 \cdot 57$ \\
\hline Patients admitted with community onset bloodstream infections & $17(3 \cdot 7)$ & $15(2 \cdot 6)$ & $0 \cdot 35$ \\
\hline \multicolumn{4}{|l|}{ MRSA surveillance and environmental contamination } \\
\hline Environmental contamination & $9 / 474(1 \cdot 9)$ & $7 / 500(1 \cdot 4)$ & $0 \cdot 53$ \\
\hline Adjacent patient room & $3(1 \cdot 3)$ & $2(0 \cdot 8)$ & $0 \cdot 59$ \\
\hline Common areas & $6(2 \cdot 5)$ & $5(2 \cdot 0)$ & 0.69 \\
\hline \multicolumn{4}{|l|}{ Outcomes of ICU stay } \\
\hline MRSA acquisition & $2(0 \cdot 4)$ & $2(0 \cdot 5)$ & $0 \cdot 93$ \\
\hline Death & $52(11 \cdot 5)$ & $27(6 \cdot 5)$ & $\mathbf{0 \cdot 0 1}$ \\
\hline Infection/sepsis-associated deaths & $25(5 \cdot 5)$ & $14(3 \cdot 3)$ & $0 \cdot 12^{*}$ \\
\hline Sepsis-associated deaths $(\operatorname{LOS}>72)$ & $11(2 \cdot 4)$ & $7(1 \cdot 7)$ & $0 \cdot 43^{*}$ \\
\hline Nosocomial infections & $11(2 \cdot 4)$ & $6(1 \cdot 4)$ & $0 \cdot 29$ \\
\hline Nosocomial CLA-BSI & $1(0 \cdot 2)$ & $1(0 \cdot 2)$ & $0 \cdot 95$ \\
\hline Nosocomial BSI & $5(1 \cdot 1)$ & $2(0 \cdot 5)$ & $0 \cdot 30$ \\
\hline Nosocomial UTI & $4(0 \cdot 8)$ & $3(0 \cdot 7)$ & $0 \cdot 79$ \\
\hline Nosocomial VAP & $1(0 \cdot 2)$ & 0 & $0 \cdot 30$ \\
\hline
\end{tabular}

* Values were calculated in a post-hoc analysis after an unexpected difference in mortality was noted in the primary analysis. CHG, chlorhexidine; MRSA, methicillin-resistant Staphylococcus aureus; ICU, intensive care unit; s.D., standard deviation; VRE, vancomycin-resistant Enterococcus spp.; C. diff, Clostridium difficile; LOS, length of stay; CLA-BSI, central lineassociated bloodstream infection; BSI, bloodstream infection; UTI, urinary tract infection; VAP, ventilator-associated pneumonia.

impending move of all ICUs to a new hospital tower. This limitation means that our findings are subject to seasonal and natural variation in MRSA and other infections [18, 19], and some evidence of this was evident. The number of contact precaution days dropped by only 139 days, due to an increase in the number of enteric pathogen-associated contact precaution days. Enteric pathogen colonisation status (e.g., VRE and C. difficile) is tracked independently from MRSA colonisation status at this centre. While it is possible that widespread use of chlorhexidine may have induced a rise in VRE and $C$. difficile, this phenomenon has not been seen in the larger clinical trials of daily chlorhexidine bathing [12-14]. We suspect our observation is a chance occurrence related to differences in admitted patients and perhaps patterns of antibiotic use.
Similarly, mortality was lower in the chlorhexidine period than the contact precautions period $(6 \cdot 5 \% \mathrm{vs}$. $11.5 \%, P=0.01)$. We also saw trends towards improved outcomes from all sepsis/infection-related deaths and sepsis/infection-related deaths $>72 \mathrm{~h}$ after ICU admission. While it is possible that chlorhexidine bathing improved infection-related outcomes, this does not seem likely in such a small and uncontrolled clinical observation. More likely the differences in mortality and infection-related outcomes were the result of random chance, differences in severity of illness, and/or the patient mix admitted to the ICU in each time period. To better understand the impact of chlorhexidine bathing on VRE, $C$. difficile, mortality and infection-related outcomes, a larger trial with a longer observation period is needed. 
Our study was further limited by being conducted in a single medical centre. Notably, the study centre had high levels of hand hygiene compliance, and relatively compliant effective environmental services, as measured by objective surveillance. Furthermore, there were only two MRSA acquisitions in the baseline period, suggesting that MRSA transmission was not a significant problem at this institution. Future investigations should be multicentre-based investigations, ideally in facilities with a higher burden of MRSA transmission to measure the effects of this policy change.

We highlight that the use of environmental contamination as an outcome variable is relatively novel and may provide a more sensitive indicator for the need for contact precautions in the healthcare setting. Future research and larger trials should consider measuring environmental contamination which likely plays an important role in pathogen transmission in the hospital setting $[10,17,20]$.

In summary, the initiation of contact precautions for patients with MRSA carriage is widely recommended [1-3]. However, in an era of increasing use of chlorhexidine as part of routine daily skin cleaning, the need for contact precautions for patients with MRSA may not be compelling or data driven. Findings from our study suggest that contact precautions might be safely discontinued in a context of universal chlorhexidine bathing. However, prior to advocating for widespread policy change in hospitals nationwide or worldwide, more comprehensive and multisite investigations, ideally done in a randomised fashion, are needed.

\section{SUPPLEMENTARY MATERIAL}

The supplementary material for this article can be found at https://doi.org/10.1017/S0950268817001121

\section{ACKNOWLEDGMENTS}

The authors greatly appreciate the Infection Control Department at Torrance Memorial Medical Center for their work in ensuring the completion of this investigation. They appreciate the efforts of the Torrance Memorial Medical Staff and the Hospital Administration to facilitate completion of this investigation. J.A.M. received support from the NIH/NCRR/ NCATS UCLA CTSI (Grant number KL2TR000122). J.A.M., S.S.H., and L.G.M. received support from the AHRQ (Grant number RC4AI092327). S.S.H. conducts clinical studies and trials in which participating hospitals and nursing homes receive contributed products from Sage Products, Molnlycke, $3 \mathrm{M}$ and Clorox. JM, L.G. M., and JM conduct clinical studies and trials in which participating hospitals and nursing homes receive contributed products from Sage Products, $3 \mathrm{M}$ and Clorox. No other authors have any conflicts of interest to disclose in relation to this manuscript.

\section{DECLARATION OF INTEREST}

None.

\section{REFERENCES}

1. Muto CA, et al. SHEA guideline for preventing nosocomial transmission of multidrug-resistant strains of Staphylococcus aureus and enterococcus. Infection Control and Hospital Epidemiology 2003; 24: 362-386.

2. Coia JE, et al. Guidelines for the control and prevention of methicillin-resistant Staphylococcus aureus (MRSA) in healthcare facilities. Journal of Hospital Infection 2006; 63(Suppl. 1): S1-S44.

3. Siegel JD, et al. Management of multidrug-resistant organisms in health care settings, 2006. American Journal of Infection Control 2007; 35(10 Suppl. 2): S165-S193.

4. McKinnell JA, et al. Cost-benefit analysis from the hospital perspective of universal active screening followed by contact precautions for methicillin-resistant Staphylococcus aureus carriers. Infection Control and Hospital Epidemiology 2015; 36: 2-13.

5. Huskins WC, et al. Intervention to reduce transmission of resistant bacteria in intensive care. New England Journal of Medicine 2011; 364: 1407-1418.

6. Harris AD, et al. Universal glove and gown use and acquisition of antibiotic-resistant bacteria in the ICU: a randomized trial. Journal of the American Medical Association 2013; 310: 1571-1580.

7. Morgan DJ, et al. The impact of contact isolation on the quality of inpatient hospital care. PLoS One 2011; 6: e22190.

8. Morgan DJ, et al. The effect of contact precautions on healthcare worker activity in acute care hospitals. Infection Control and Hospital Epidemiology 2013; 34: 69-73.

9. Morgan DJ, et al. Adverse outcomes associated with contact precautions: a review of the literature. American Journal of Infection Control 2009; 37: 85-93.

10. Hardy KJ, et al. A study of the relationship between environmental contamination with methicillin-resistant Staphylococcus aureus (MRSA) and patients' acquisition of MRSA. Infection Control and Hospital Epidemiology 2006; 27: 127-132.

11. Price JR, et al. Whole-genome sequencing shows that patient-to-patient transmission rarely accounts for 
acquisition of Staphylococcus aureus in an intensive care unit. Clinical Infectious Diseases 2014; 58: 609-618.

12. Chen W, et al. Effects of daily bathing with chlorhexidine and acquired infection of methicillin-resistant Staphylococcus aureus and vancomycin-resistant Enterococcus: a meta-analysis. Journal of Thoracic Disease 2013; 5: 518-524.

13. Huang SS, et al. Targeted versus universal decolonization to prevent ICU infection. New England Journal of Medicine 2013; 368: 2255-2265.

14. Climo MW, et al. The effect of daily bathing with chlorhexidine on the acquisition of methicillin-resistant Staphylococcus aureus, vancomycin-resistant Enterococcus, and healthcare-associated bloodstream infections: results of a quasi-experimental multicenter trial. Critical Care Medicine 2009; 37: 1858-1865.

15. Hibbard JS, Mulberry GK, Brady AR. A clinical study comparing the skin antisepsis and safety of ChloraPrep, 70 isopropyl alcohol, and 2 aqueous chlorhexidine. Journal of Infusion Nursing 2002; 25: 244-249.
16. Horan TC, Andrus M, Dudeck MA. CDC/NHSN surveillance definition of health care-associated infection and criteria for specific types of infections in the acute care setting. American Journal of Infection Control 2008; 36: 309-332.

17. Cimolai N. MRSA and the environment: implications for comprehensive control measures. European Journal of Clinical Microbiology \& Infectious Diseases 2008; 27: 481-493.

18. Mermel LA, Machan JT, Parenteau S. Seasonality of MRSA infections. PLoS One 2011; 6: e17925.

19. Gilca R, et al. Seasonal variations in Clostridium difficile infections are associated with influenza and respiratory syncytial virus activity independently of antibiotic prescriptions: a time series analysis in Quebec, Canada. Antimicrobial Agents and Chemotherapy 2012; 56: 639-646.

20. Boyce JM. Environmental contamination makes an important contribution to hospital infection. Journal of Hospital Infection 2007; 65(Suppl. 2): 50-54. 